\title{
PRACTITIONER BUY-IN AND RESISTANCE TO E-ENABLED INFORMATION SHARING ACROSS AGENCIES
}

The case of an e-government project to join up local services in England

\section{SUSAN BAINES, PAT GANNON-LEARY AND ROB WILSON}

Centre for Social and Business Informatics, University of Newcastle-upon-Tyne, Newcastleupon-Tyne, UK

Abstract: FrAmework for Multi-agency Environments (FAME) is one of 23 national projects within the e-government strategy to reform and modernize local services in England. Six local projects each worked with an IT supplier (known as a technology partner) to produce a technical system for the exchange and management of client / patient information across agency and professional boundaries. All participants, including the technology partners, insisted that FAME was about people, organizations and change more that it was about technology. This paper draws upon the successes and setbacks of these local projects in order to report some urgent lessons for the implementation of e-government initiatives that involve new working practices for front-line practitioners.

Key words: e-social care; local government; front-line practitioners; ICT project evaluation

\section{JOINING UP LOCAL GOVERNMENT SERVICES}

Numerous policies and initiatives in the UK now aim to make public services provided by different agencies more efficient, responsive and 'joined-up'. 'Joining up' has come to denote ways in which the New Labour government has reacted to the perception that complex social needs demand co-coordinated activities across organizational boundaries (Ling 2002). For example, the National Service Framework (NSF) for older people sets out standards which aim to promote older people's health and independence and 
ensure that services for them are joined-up and tailored to their needs. Thirty five English local authorities were awarded the status of 'Pathfinder' Children's Trusts in summer 2003. Their remit is to co-ordinate local education, social care and some health services for children and young people. The Children Act 2004 now makes it incumbent on all education, health and social service providers to work together to deliver better services focused on the child.

Fragmentation of agencies in social care and health has been blamed for poor service, inefficiency, and failures of care. A tragic instance of failure of care was the case of Victoria Climbié - an eight year old girl killed by her guardians despite being known to several agencies and services:

Victoria Climbié came into contact with several agencies, none of which acted on the warning signs. No one built up a picture of her interactions with different services (DfES 2003).

In other words professionals who had contact with this vulnerable child failed to protect her at least partly because they did not share information they individually held.

FrAmework for Multi-agency Environments (FAME) was designed to develop a framework for sharing personal information between local authorities and other agencies. The image of the organizational and professional 'silo', in which information is inert, is ubiquitous in UK government policy documents and has come into wide usage. From within their 'silos' service workers are able to see only one aspect of an individual who may have complex needs. 'Joining up' across the silos requires personal information about users of services to be made available across organizations and agencies (including statutory bodies, voluntary groups and for-profit service providers) with different cultures, management structures, and information systems.

FAME was one of the largest and most ambitious of the national projects created to support the delivery of local e-government in England. Within FAME there were six work strands each led by an English local authority in partnership with service providers. From April 2003 to October 2004 these six local FAME projects were each required to deliver a real life example of e-enabled information sharing across agencies in a particular set of services (for example, to vulnerable older people, mental health patients, disabled children). Each strand worked with an IT supplier known as a 'technology partner'. All the strands involved Social Services. There were partners in each case from some (but not necessarily all) of the following: Health, Education, the Police, voluntary sector agencies, and other local authorities. In developing the local solutions the technology partners visited practitioners in their workplaces and held workshops with them in order to ensure that the functionality and the 'look and feel' met their needs. Each technical solution 
was different, reflecting local and service specific conditions and priorities. All had the remit to link participating agencies and their IT systems in order to facilitate the secure and timely exchange of information according to locally agreed protocols. We will use two examples of local FAME projects here for illustration, an electronic Single Assessment Process (SAP) for vulnerable older adults and a 'virtual integrated mental health record'.

The aim of the SAP project was to deliver a working electronic Single Assessment Tool in order to improve the way older people are jointly assessed for their health, social care and housing needs. Embedded within the electronic tool is a Department of Health accredited assessment instrument which can also be used in paper form. The SAP application allows practitioners across all participating agencies to assess the needs of elderly people by the use of the electronic version of the assessment instrument. Practitioners can then refer cases on for further, more in depth assessment electronically. Assessments are viewed via an internet browser. The information collated as a result of these assessments is fed into an 'overview assessment summary' to give a complete, holistic picture of that elderly person's needs and involvement with other agencies. Service users are asked for consent to their information being passed on to specific agencies and data accessibility is restricted to match this consent.

The FAME virtual integrated mental health record was developed across two neighboring local authority areas where community mental health teams (social care and health workers) had been integrated for more than 10 years and an integrated paper record was already in use. Electronic records, however, were still held on separate systems. The incentive to participate in FAME was described by the service manager as making technology 'catch up' with existing practice so that providers would present a seamless service to the users. This was not happening because the paper file resided with the main team dealing with the service user. Records were transported between providers across the county by courier. Liaison between teams was by telephone, email and fax and service users were likely to be asked for information they had already supplied to another professional. The FAME virtual integrated mental health record project produced an application that enables practitioners in two pilot sites to read information about service users from the existing core operational systems of the local authority and health partners. They can see names, aliases, current and previous addresses, contact numbers, and lists of when referrals have been made, by whom and to whom.

Summaries of each local strand and some of the supporting products including technical statements of requirements and integration specifications are now in the public domain. They are available from the FAME website http://www.fame-uk.org/about/strand/ as exemplars for the benefit of other 
local authorities and their partners. This article does not duplicate these details. It focuses upon just one key aspect of the development and implementation of local, electronic information sharing solutions for joinedup working - the responses of professional workers in the participating agencies. Unlike the technical, ethical and legal issues around information sharing this is a relatively undeveloped theme in the policy or academic literature. Yet it affects how government policy does, or does not, get translated into practice.

In addition to the six local authority led projects (known as strands) already referred to, FAME had two further strands: The Generic Framework and Learning \& Evaluation - both led by a Newcastle University team of which all the authors were part. The Generic Framework identified and described nine building blocks that are essential to effective multi-agency working. (See http://www.fame-uk.org). The Learning \& Evaluation team worked closely with the six local strands, exploring factors that contributed to successful delivery. This article is based upon data collected for the Learning \& Evaluation strand.

Overall the evaluation of the FAME local strands was positive despite setbacks beyond the control of the local teams that led to delays in implementation (Baines et al. 2004). One of FAME's key achievements is the wealth of evidence it provides that local authorities and their partners can create multi-agency environments in which information is made accessible electronically to practitioners across traditional service boundaries. Indeed, some practitioners reported that they were able to see the 'whole' patient /client in ways that had not previously been possible. Very importantly, FAME delivers information that they value. Yet three months after new IT systems 'went live' in the two strands that implemented them on schedule, overall levels of system usage were low. The article draws upon these struggles within FAME in order to identify some urgent lessons for egovernment initiatives that involve front-line professionals.

First we put the FAME experience in context by overviewing literature that has offered insight into social care and health practitioners' responses to multi-agency initiatives and IT. Then we introduce the empirical research part of the evaluation of FAME - and describe the research methods. We give some more details about the FAME projects and report and comment upon what we learned about practitioner attitudes, experiences and behavior. Quantitative data from across FAME is reported but for reasons of space we concentrate upon qualitative material from the strands that worked with the services for vulnerable older people and mental health. Finally we reflect upon this material to point to lessons and to make recommendations for egovernment projects. 


\section{BUY-IN OR RESISTANCE?}

The theme of 'joined-up' or 'holistic' public services is intimately associated with the modernization agenda of the New Labour government in the UK; but it is not new (6 et al. 2002; Pollitt, 2003). There is long history of joint endeavor based on shared planning, co-location of services and other physical means of attempting to promote more co-ordinated public policy and policy delivery. What is new is the scale of ambition of the contemporary efforts in at joining up at the level of policy implementation and service delivery. New confidence in the possibility of such joining up is substantially based on the claimed powers, and in particular the integrating capacity, of new information and communication technologies (Hudson, 2003; Geoghegan et al., 2004).

Workers who interact directly with citizens in the delivery of public services implement government policies. We refer to these people (social workers, health workers, police officers, teachers) as 'front-line practitioners'. They are the group labeled by Lipsky (1980) as the 'street level bureaucrats' through whom most citizens encounter government and whose actions constitute the services delivered by government. FAME put information systems in place at a local level in order to support the flexible and person-centered approaches now demanded of service providers in health and social care. Our participation as researchers in FAME afforded a unique opportunity to explore encounters by front-line public sector workers with IT-enabled change. As a result we have been able to open up this neglected element of the broader e-government agenda.

We look next at some evidence from earlier research on joining up in social care and health and why it is so hard to achieve at the level of frontline service delivery. Then we turn more specifically to IT and suggest selectively some approaches from a much wider literature on IT and professional working practices that can help to contextualize the experiences of social care and health workers in e-government initiatives.

Formal mechanisms put in place by agencies at a strategic level do not necessarily produce the intended cooperation on the front-line (Lupton, 2001). This may be because workers in participating agencies are not fully aware of the needs, limitations and pressures of the others (Payne et al 2002). Another practical factor is lack of time to develop relationships within project timescales (Atkinson et al. 2001). Attempts to create multiagency information systems (whether paper or computer based) have often failed as a result of different 'mindsets', in particular different attitudes towards the recording, storage and distribution of information (Green et al. 2001). Professionalism may be perceived as under threat (Secker and Hill 2001). Such intractable barriers to multi-agency working are repeatedly 
labeled 'cultural'. Policy documents from central government in the UK repeatedly demand the dismantling of service 'silos' through cultural change. For example, the Green Paper Every Child Matters stated that local authorities are required to lead a process of 'cultural change'; new technologies for sharing information, according to this document, must be adopted but this alone will not bring about intended reforms towards more joined-up working practices (DfES 2003). The National Service Framework (NSF) for children similarly calls for a 'cultural shift' resulting in services being designed and delivered around the needs of children and families (Department of Health 2004).

The Green Paper (DfES 2003) cited above presents the sharing of personal information about citizens among the agencies that work with them as both desirable and inevitable in order to deliver benefits to individuals, families and society. Yet legal commitments to the protection of privacy are potentially in conflict with this agenda (6 et al. 2005). Exchanging personal data raises a wide range of issues about privacy and the balance between individual rights and the common good (Performance and Innovation Unit 2002). In practice tensions between information sharing and the protection of privacy are usually addressed by the use of safeguards in the form of detailed guidelines (Bellamy et al. 2005). Front-line practitioners are required to interpret such guidelines and incorporate them into their practice.

Professional expertise and IT can come into conflict on many levels. Professional workers emphasize the complex, contextual nature of front-line activities; they sometimes perceive the introduction of IT into their work as undermining their expertise and replacing it with a standardized labor process characterized by centralization of control (Haynes 2003). It has been argued that the professional care and health worker is losing authority to the citizen 'expert' as well as to the control of the state through processes of ever greater 'informatization' (Nettleton, 2003; Harrison, 2002). At the same time some reports have found that front-line practitioners believe that the caring and relational aspects of their work are threatened. One study, for example, reported that midwives saw an IT system for recording patient information as antithetical to the 'woman-centered' values of their profession (Henwood and Hart, 2003). Seemingly irrational resistance to the introduction of new technology can became understandable when examined in the light of workplace histories of technology use and earlier experiences that may have challenged workers' self image and professional relationships (Stam et al 2004).

In summary: Information systems have a vital role to play in enabling the access to timely, accurate and trusted information that is essential for joinedup working but they are likely to be only part of the solution. Other ingredients in recipes for reform are 'cultural change' (which is usually ill 
defined) and sets of instructions, protocols and guidelines likely to add to the ever increasing complaint of information overload. Moreover, there is evidence that from the perspectives of some front-line professional workers new information systems are not a solution at all but a threat.

\section{EMPIRICAL RESEARCH: UNDERSTANDING CHANGE IN THE WORKPLACE FOR PRACTITIONERS}

The overarching aim of the FAME Learning \& Evaluation strand was to draw upon the experiences of the local projects in order to document, assess and report what worked, what did not work, and why. Evaluation is conventionally divided between 'summative' (to determine overall effectiveness) and 'formative' (giving feedback to people trying to improve an intervention) (Newburn 2001). There is blurring at the edge however and some commentators maintain that the distinction is often exaggerated. Our work cut across these modes with emphasis on the formative. We were guided by the principles of Theory of Change (Connel and Kubisch 1998). Central to a Theory of Change evaluation is the requirement that the evaluator works to surface the implicit theory (or theories) of action held by all participants.

The FAME Learning \& Evaluation team undertook field work from July 2003 to October 2004. We consulted project managers, project board chairs and a wide range of stakeholders including service managers, service user representatives, and front-line practitioners. We undertook the following activities:

- Meetings with project managers;

- Meetings with project partners and stakeholders;

- Observation of local events, meetings, and workshops;

- Document analysis;

- Visits to pilot sites;

- A questionnaire survey and interviews with front-line practitioners;

- Report back to project teams.

All the local project teams informed us at our first meetings that 'buy-in' from practitioners was both essential and fraught with difficulty. Project managers and others typically expressed this concern in words to the effect that 'the technology will be easy - the real challenge will be changing the ways people work - changing culture'. They feared that hard pressed health care/social workers would simply 'see it as more work'. Practitioners, we were told, get blasé and weary and often suffer from 'project fatigue'. In 
some instances practitioners were struggling with the implementation of other new processes and systems in parallel with the FAME project. One project manager explained that she was 'dealing with reluctance and resistance.' Again and again, project managers and other team members highlighted lack of practitioner 'buy-in' as a serious risk factor. In other words their 'theories of change' were underpinned by the perception that practitioner 'resistance' must be addressed in order to ensure that the potential benefits of the projects would be realized. That is why we devoted time and resources in our evaluation to activities (observations, questionnaires and interviews) designed to elicit the experiences of practitioners across professions and agencies.

Questionnaires for practitioners prior to implementation were designed by the Learning \& Evaluation team after the initial round of meetings with project managers and observation of some early work with practitioners in the strands. They were distributed to practitioners in the pilot sites by the project teams in four strands. (In two strands this was not possible because of delays in identifying which agencies and staff would participate.) The timing of this questionnaire was such that practitioners had been exposed to the aims and objectives of FAME from publicity in the workplace and from local awareness-raising events but none had yet been trained to use the system. Overall we received 108 pre-implementation questionnaires from practitioners who had been selected by project teams to be trained to use the FAME IT systems.

Response rates for the questionnaire from individual locations were variable. They ranged from an excellent 60 per cent in one strand to below 10 per cent in another. The qualitative and quantitative data we collected from practitioners prior to the implementation of FAME IT systems in four strands offer insight into attitudes, perceptions and resources that facilitate or impede multi-agency-environments and IT use. We were able to do some post-implementation evaluation work in the two strands that 'went live' in summer 2004. We also benefited from access to some local evaluation work conducted by one of the project teams. As well as a new questionnaire and interviews by telephone with selected respondents we observed postimplementation events and meetings organized by the strands.

\section{4. "PASSING THE PAIN BARRIER": PRACTITIONERS' EXPERIENCES AND RESPONSES}

The evidence from the pre-implication questionnaire was that practitioners who had been introduced to FAME generally understood and 
supported its aims. Very importantly, they recognized that lack of coordination and exchanging information across agencies leads to less than optimal services to clients / patients.

- More than four fifths (82.5 per cent) of respondents agreed that lack of information sharing caused poor outcomes;

- More than two thirds (70 per cent) of respondents agreed that they relied on service users for information about other agencies/services;

- More than four fifths ( 84 per cent) of respondents agreed that increased knowledge of the work of other agencies/services would benefit their service users;

- Similarly, 86 per cent of respondents agreed that working more closely with other agencies/services would benefit their users.

Three quarters of respondents described themselves as regular IT users. Nevertheless, the prior IT experience and skills of practitioners, and their access to IT, were extremely variable. In some cases both skills and access were low. (Indeed, as later qualitative work revealed, this was a practical barrier to participation in an IT initiative.) More than two out of five respondents (42.3 per cent) reported that they lacked exclusive access to a PC in their workplace.

Only just over a third ( 37 per cent) indicated that they were unsure what information they were allowed to share with other agencies/services. Nearly half ( 47.5 per cent) indicated that they currently shared information with individual representatives of other agencies/services on an informal basis. Nevertheless, more than three quarters of all respondents ( 76 per cent) agreed that clearer guidelines on sharing information would be helpful to them. In respect of potential deterrents to sharing information, 45 per cent of respondents indicated that Data Protection issues deterred them, while 56 per cent were deterred by issues around client consent and confidentiality. Practitioners were asked to respond in their own words to the question 'What, in your view, are the main barriers to sharing information with other agencies/services?'

The most frequently cited responses were:

- Data Protection issues, lack of knowledge re legality, fear of litigation or of disciplinary action ;

- Lack of contact with known (knowledgeable) individuals, access to appropriate people at the right time;

- Lines of communication, different systems, delays;

- Lack of time;

- Confidentiality issues, protocols, not knowing how much to say; 
- Lack of information about other agencies and services involved with clients/patients.

These findings can not of course be claimed as representative statistically of the wider population of care and health workers in the UK who are, or will become, affected by e-government initiatives. Nevertheless they are indicative of: positive attitudes to the 'joining-up' agenda; a perception that information sharing is necessary but difficult; and unevenness of IT skills and resources.

Questionnaire data were supplemented by observation of meetings, events and workshops at which practitioners were present. For example, we sat in on a selection of the workshops run by the IT partners for practitioners. In general practitioners were interested and enthusiastic about the promise of an electronic system to improve the quality and timeliness of information. Some practitioners, however, expressed anxiety that the IT system would reduce personal contact and trust. In one workshop for Health professionals, for example, it was pointed out that, where there is a history of face-to-face relationships, practitioners know a person and what s/he will do with the information. Comments made by practitioners in the workshops we observed confirmed the questionnaire evidence for shortfall in IT resources and skills. For example, school nurses reported that they had one PC between 15 . Community nurses said that six of them shared a PC which crashed at least once a day. One nurse commented with heavy irony, 'my IT skills are improving every day - I now use two fingers!'

Late in the process we observed a workshop for practitioners led by the technology partner in the strand that was working towards the creation of an electronic single assessment process (SAP) for vulnerable older people. Earlier workshops had been for practitioners in specific services but this one included a mixture of health and social care workers. One of the most interesting and positive features was the interaction between the practitioners as they discussed their different practices and attitudes to service users' information. Some seemed surprised at what they heard from practitioners in other professions. For example, a district nurse explained that she always left her records with patients in their homes. A social worker commented that he would never leave any record with a client and asked her why she did so. One reason, she said, was security - it is not safe to keep confidential records in a car between visits. Another reason was to 'empower' patients - 'it is the patient's record'. This dialogue continued for some time. This was a reciprocal exchange of ideas about practice across agencies. It helps to confirm the inference from the questionnaire results that practitioners, in principle, value increased knowledge of the work of other agencies.

Our post-implementation work was limited to two FAME strands which had an IT system in use by summer 2004. There was (1) the virtual, 
integrated mental health record project and (2) the single assessment process (SAP) for vulnerable older people. SAP was atypical in FAME in that it included two separate (but co-operating) sites, one in the north of England and one in the south. The SAP strand involved by far the largest number of practitioners ( 80 in the southern site and 130 in the northern one). Unlike the virtual integrated mental health record, both the SAP systems included the facility for practitioners to write information in as well as read it.

After implementation of SAP some practitioners were extremely enthusiastic about the capacity of the new IT system to reveal the 'whole picture' of a patient/client. A psychiatric liaison officer in a hospital, for example, reported an early case where he had seen positive benefit for a patient. An elderly man had come into Accident and Emergency (A\&E) with apparent memory problems but an assessment of him completed earlier gave a picture which showed that this was a result of medication and not a case of dementia. Without this assessment information A\&E staff would have taken the memory loss at face value. Further positive comments from free texts answers in a questionnaire sent out after implementation in the northern SAP site included:

- '[I was] unsure about FAME to start with but as I began to use it more I could see an increased benefit for both patient and carer'

- 'When I have logged onto FAME as a duty enquiry to our department $I$ found the information available really useful and comprehensive.'

- '[I was] able to print out an overview assessment which was completed by the Health Visitor [the] information shared led to agreed joint planning and care services...'

Overall, however, practitioners who had the opportunity were slow to adopt the system. The northern SAP site (which had trained the largest number of practitioners in any part of FAME) found that just under a third of them were using the system in any way three months later.

In order to understand and address the problem of low usage the northern SAP project team invited practitioners to a 'review day' in August 2004 in a local hotel. The Project Board Chair introduced the first session by saying, 'the steering group has gone through the pain barrier but practitioners are still in pain!' Practitioners were asked to articulate their concerns and barriers to using the system. The main points they made were:

- This is just another project - it will not last.

- Uncertainty over the IT strategy of the National Health Service discourages buy-in.

- It takes time to use the system and taking that time means giving a worse service and imposing burdens on colleagues. 
- It is not easy to see direct benefits for clients/ patients from using an IT system when immediate concerns are about finite resources and expanding need. 'I worry that we will have a fantastic electronic system and no service to give people!'

Some practitioner groups are expected to put in information - at the cost of their time and effort - but will not benefit from receiving it. Some claimed that using SAP will 'punish' them. A social worker - one of the most enthusiastic SAP users - observed that the heart of the problem of low usage by colleagues is that a new 'user perspective' is needed. When you put information into SAP it benefits someone else such as an NHS worker in the hospital - 'but we must see the big picture - we are all one team'. The idea of harnessing the commitment of such individuals to animate wider interest in SAP was suggested as a way forward.

An internal evaluation questionnaire sent out to practitioners by the SAP project teams in both sites sought opinions about improvements in working practices since the introduction of SAP. In some instances double entry of data had been necessary and this, together with inexperience on the new systems, had at times slowed things down. Whilst most practitioners indicated that SAP was helping to develop 'new ways of working', there was less certainty about improving speed of access to services. These differences may be attributable to participants' particular settings (e.g. the number of persons sharing one $\mathrm{PC}$ as indicated in the pre-implementation survey results) as well as the project's limited scale and other factors outside of its control. On a positive note, responses to the local strand questionnaires demonstrated that trust between workers in partner organizations had improved, and 'a common language' was developing.

The FAME strand that developed a virtual integrated record for mental health also suffered from slow uptake by health and social care practitioners. This project trained 30 staff across two pilot sites and 'went live' in May 2004. When we visited one of these sites in late September 2004 the manager informed us that 13 individuals in his team had to date been trained to use the IT system but only six of them regularly did so. He attributed low usage to early technical difficulties they had experienced with access onto the system. Of the eight practitioners from that strand who responded to our post-implementation questionnaire six answered an open ended question about barriers to using FAME with reference to technical problems, for example, 'system failure, denied access' and 'time, reliability and speed of access'. Many of the practitioners nevertheless remained optimistic that in the longer term the virtual integrated mental health record will have value for them and their service users. In telephone interviews they told the Learning \& Evaluation team that they liked having relevant information at 
their fingertips and appreciated the fact that they no longer have to wait for patients' notes to arrive on paper.

The most intractable barrier to usage of FAME IT systems is the one highlighted in the 'review day' in the northern SAP site referred to above. Putting information onto the system is perceived to represent a cost in time and resources for which there is no obvious payback. From this evidence the inequality of costs and benefits for individual practitioners and agencies appears to be a serious obstacle to the successful introduction of information systems for multi-agency working.

It was not possible to undertake post-implementation work with practitioners in the stands that did not 'go live' with their IT systems until autumn 2004. It would have been particularly valuable to contrast, in the longer term, practitioner attitudes and responses in one of these local strands, where their input into defining and designing the system was more intensive than in those discussed above. Because this could not be included in our analysis some valuable potential learning was lost.

\section{CONCLUSIONS}

Our role as researchers in the Learning \& Evaluation strand of FAME gave us excellent access to front-line practitioners participating in the implementation of an e-government programme. Evaluation inevitably makes demands on the evaluated (Draper 2001). We were extremely fortunate in the level of support and co-operation we received from the local project teams. Our evaluator role, however, was also a limitation in that it was tied to the time frame of an 18 month programme that suffered delays in implementation. Post-implementation evaluation was possible only in two of the six local strands. This is regrettable because local conditions, and the strategies adopted by the project teams, varied across the strands. A longer period between implementation and final reporting would have been needed in order to refine and maximize learning from the achievements of all the FAME local strands.

The main lessons from the evaluation of FAME with regard to front-line practitioners are:

- Nothing should be assumed about IT access and skills. There were serious limitations in some service providers' IT resources, as indicated in the questionnaire responses and workshops discussed above. The IT experience and skills of practitioners were extremely uneven. New projects would benefit from an early audit of IT skills and resources. 
- Some practitioners complained of technical frustrations yet remained optimistic about the potential benefits of IT enabled information sharing. This evidence suggests that they can be extremely resilient to practical set-backs in technological performance when the idea of improved working practice has taken root.

- Workshops that brought together front-line practitioners from different professions and agencies stimulated interest and dialogue, inspired by a common interest in better and more timely information. There was no evidence that cultural differences per se between these groups prevented multi-agency working.

- There were however practitioners who reported that they were reluctant to become users of FAME because they saw no direct rewards for their own practice - although they recognized that other agencies may benefit. The perceived inequality of costs and benefits needs to be anticipated and managed.

- Recruiting enthusiastic and committed practitioners as 'super users' to help overcome resistance from others is one tentative solution for low practitioner uptake.

- It was often re-iterated by participants - including the technology partners - that FAME was not about IT but about people, organisations and culture. Some claimed that others (mistakenly) think it is about technology. It is our judgment (based on evidence from within and beyond the FAME strands) that the technology / practice dichotomy is misleading. Multi-agency practice and IT should be understood as two facets of the same whole.

\section{REFERENCES}

6, P., Bellamy, C. and Raab C. 2005 Joined-up government and privacy in the United Kingdom: managing tensions between data protection and social policy, Part I, Public Administration, 83 (1) $111-133$.

6, P., Leat, D., Steltzer, K. and Stoker, G. 2002 Toward Holistic Governance: the new reform agenda, Basingstoke: Palgrave.

Atkinson, M., Wilkin, A., Stott, A. and Kinder, K. 2001 Multiagency Working: an audit of activity, Slough: The National Foundation For Educational Research.

Baines, S., Gannon-Leary, P. and Walsh, S. 2004. FrAmework for Multi-Agency Environments (FAME): Final Report of the Learning \& Evaluation. http://www.fameuk.org/about/strand/

Bellamy, C., 6, P. and Raab, C. 2005. 'Joined-up government and privacy in the United Kingdom: Managing tensions between data protection and social policy. Part II.' Public Administration 83(2): $393-415$.

Connell J P and Kubisch A C 1998. Applying a Theory of Change Approach to the Evaluation of Comprehensive Community Initiatives: Progress, Prospects and Problems, Aspen Institute, USA. 
Department of Health 2004. NSF for Children, Young People and Maternity Services, London, Department of Health.

DfES 2003 Every Child Matters, HMSO, London.

Draper, L. 2001. 'Being evaluated: a practitioner's view', Children \& Society 15(1): 46 - 52

Geoghegan, L., Lever, J. and McGimpsey, I. 2004. ICT for Social Welfare: A toolkit for managers. Bristol, The Policy Press.

Green, A., Maguire, M. and Canny, A. 2001 Keeping Track: Mapping and tracking vulnerable young people, Bristol, The Policy Press.

Harrison, S. 2002. 'New Labour, modernisation and the medical labour process.' Journal of Social Policy 31(3): 465-485.

Haynes, P. 2003. Managing Complexity in the Public Services, Maidenhead, Open University Press.

Henwood, F. and Hart, A. 2003. 'Articulating gender in the context of ICTs in health care: the case of electronic patient records in the maternity services.' Critical Social Policy 23(2): 249-267.

Hudson, J. 2003. 'E-galitarianism? The information society and New Labour's repositioning of welfare.' Critical Social Policy 23(2): 268-290.

Ling T. 2002. 'Delivering joined-up government in the UK: dimensions, issues and problems', Public Administration, 80 (4): 615-642.

Lipsky, M. 1980. Street Level Bureaucracy, New York, Russell Sage Foundation.

Lupton, C., North, N. and Khan, P. 2001. Working Together or Pulling Apart? The National Health Service and child protection networks. Bristol, The Policy Press.

Nettleton, S. and R. Burrows 2003. 'E-Scaped Medicine? Information, Reflexivity and Health.' Critical Social Policy 23(2): 165 - 185.

Newburn, T. 2001 'What do we mean by evaluation?' Children \& Society 15(1): 5 - 13.

Payne, S., Kerr, C., Hawker, S., Hardey, M. and Powell, J. 2002 'The communication of information about older people between health and social care practitioners', Age and Ageing 31(2): 107-117.

Performance and Innovation Unit 2002. Privacy and data-sharing: the way forward for public services, London, Cabinet Office.

Pollitt, C. 2003. The Essential Public Manager. Maidenhead, Open University Press.

Secker, J. and Hill, K. 2001. 'Broadening the Partnerships: Experiences of Working across Community Agencies', Journal of Interprofessional Care 15 (4):341-350.

Stam, K., Stanton, J. and Guzman, R. 2004. 'Employee resistance to digital information and information technology change in a Social Service agency: A membership category approach', Journal of Digital Information, 5 (4): 10-20.

\section{Acknowledgement}

We are grateful to the Office of The Deputy Prime Minister (ODPM) for funding the research upon which this article is based. All opinions and interpretations are the responsibility of the authors. 